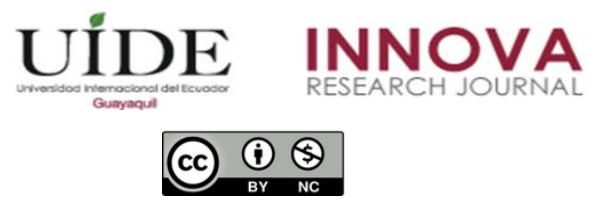

INNOVA Research Journal, ISSN 2477-9024

(Septiembre-Diciembre 2020). Vol. 5, No.3.1 pp. 15-39

DOI: https://doi.org/10.33890/innova.v5.n3.1.2020.1531

URL: http://revistas.uide.edu.ec/index.php/innova/index

Correo: innova@uide.edu.ec

\title{
Impacto de los canales de comercialización online en tiempos del COVID-19
}

\section{Impact of online marketing channels in times of COVID-19}

\author{
Jaime Daniel Tello Saldaña \\ (Dhttps://orcid.org/0000-0002-1498-6216 \\ María Alexandra Nizama Imán \\ (D) https://orcid.org/0000-0003-3616-4843 \\ Brigitte de los Ángeles Huamán Yovera \\ (1) https://orcid.org/0000-0001-6883-1482 \\ Jorge Alberto Vargas Merino \\ (D) https://orcid.org/0000-0002-3084-8403 \\ Universidad Norbert Wiener, Perú
}

Autor para correspondencia: danieltesa2593@gmail.com; anizama52@gmail.com;

gigidlngls12@gmail.com; jorgealbvarmer5@gmail.com

Fecha de recepción: 13 de septiembre de 2020 - Fecha de aceptación: 20 de noviembre de 2020

\section{Resumen}

Las empresas peruanas, específicamente en Lima Metropolitana, debido a la coyuntura del COVID-19, se encuentran inmersas en el reto de implementar opciones para generar ventas ¿Son los canales de comercialización Online, una buena alternativa de reactivación del comercio de bienes y servicios en tiempos de COVID-19? El presente trabajo de investigación tuvo como objetivo general identificar cómo los canales de comercialización online impactan en las ventas en tiempos de COVID-19 en Lima Metropolitana 2020. El enfoque de la investigación fue cuantitativo, tipo básica, de nivel explicativo, diseño no experimental y de corte transversal. La población objeto de estudio estuvo conformada por 267 habitantes de la ciudad de Lima Metropolitana, de ambos sexos, de 18 a 55 años. La técnica utilizada fue la encuesta, el instrumento fue el cuestionario, con un estadístico global de fiabilidad alfa de Cronbach de 0,912. Los resultados inferenciales de la prueba Chi cuadrado de Pearson, demuestran que los canales de comercialización online (con un nivel altamente significativo $0,00<0,05$ ) si tiene impacto en el incremento de las ventas en tiempos de COVID-19. Se identifica también que, si existe impacto entre los canales de comercialización online y la intención de compra, así también con riesgo de compra. Se concluye finalmente que, los canales de comercialización online están altamente vinculados con el nivel de gasto y con la satisfacción de compra, es decir que el uso de los canales de comercialización online si tiene un gran impacto en el nivel del gasto y la satisfacción de compra.

Palabras claves: canales de comercialización online; COVID-19; intención de compra, nivel de gasto; satisfacción de compra. 


\begin{abstract}
Peruvian companies, specifically in Metropolitan Lima, due to the situation of COVID-19, are immersed in the challenge of implementing options to generate sales. Are online marketing channels a good alternative to reactivate trade in goods and services in times of COVID-19? The general objective of this research was to identify how online marketing channels impact sales in times of COVID-19 in Metropolitan Lima 2020. The focus of the research was quantitative, basic type, explanatory level, non-experimental design and cross-sectional. The population under study consisted of 267 inhabitants of the city of Metropolitan Lima, of both sexes, from 18 to 55 years old. The technique used was the survey, the instrument was the questionnaire, with a global statistic of Cronbach's alpha reliability of 0,912. The inferential results of Pearson's Chi-square test, show that online marketing channels (with a highly significant level $0.00<0.05$ ) do have an impact on the increase of sales in times of COVID-19. It is also identified that, if there is impact between online marketing channels and purchase intention, so with purchase risk. Finally, it is concluded that online marketing channels are highly linked to the level of expenditure and purchase satisfaction, i.e., the use of online marketing channels has a great impact on the level of expenditure and purchase satisfaction.
\end{abstract}

Keywords: online marketing channels; COVID-19; purchase intention, level of spending; purchase satisfaction.

\title{
Introducción
}

Las empresas peruanas específicamente en Lima Metropolitana, debido al avance de la pandemia del COVID-19, se encuentran inmersas en el reto de implementar el comercio electrónico, como estrategia para fomentar las ventas online, buscando infundir en los compradores la confianza en este sistema, pues debido al temor de contagio y a la continuación de medidas restrictivas, se viene experimentando una creciente dependencia del uso de un conjunto de herramientas de comercialización electrónica, que ya ha reportado tendencias de incremento de hasta un $48 \%$ en la primera semana de la cuarentena según un informe de Instituto Peruano de Economía.

Esta investigación busca conocer el impacto del comercio online en las ventas durante el proceso de pandemia COVID-19, a través del uso de plataformas online por compradores sin distinción de edad, menos experimentados, que se atreven a usar de manera conveniente la tecnología, por las mismas restricciones de movimiento, y buscando la prevención de contagios, iniciando una nueva experiencia al comprar. Se busca conocer si los cambios generados por COVID-19, influyen en decisión de compra e impactarían en el nivel de las ventas de las empresas en Lima Metropolitana.

\section{Problemática}

¿Son los canales de comercialización Online, una alternativa de reactivación del comercio de bienes y servicios en tiempos de pandemia?

La aparición de la pandemia del nuevo coronavirus: COVID-19, ha vuelto incierto las grandes economías mundiales, pues se han visto en serios problemas económicos al ver paralizadas casi el $100 \%$ de sus principales actividades generadoras de divisas, la caída de los índices de las más importantes acciones globales, han reportado grandes pérdidas en los 
mercados bursátiles, pudiendo afectar los ahorros personales y los fondos de inversión de pensiones. En este contexto, el comercio electrónico, está cobrando protagonismo, en medio del avance de la pandemia, lo que significó para Amazon un incremento en sus ingresos mundiales del $33.5 \%$ en el segundo trimestre, duplicando sus ingresos netos en \$ 5,240 millones, al mismo tiempo que proyectó invertir \$ 4 mil millones de dólares en el siguiente trimestre en gastos extraordinarios relacionados con la pandemia , los cuales fueron superados a consecuencia de los costos que surgieron como gastos extraordinarios relacionados con el brote del COVID-19 (Digital Commerce 360, 2020)

Así mismo, América Latina, en casi 5 años ha logrado un gran avance en el comercio electrónico al pasar de 105 millones de compradores digitales a más de 155, con lo que pronostica que las ventas en retails de comercio electrónico en Latinoamérica pasen de 29,800 millones de dólares en 2015 a 64,400 millones en 2019, siendo importes aún muy pequeños comparados con Asia Pacifico, Europa y Estados unidos. En este contexto se destaca a Argentina como la tercera mayor población de Latinoamérica que accede a internet, por el contrario, países como México y Colombia, siendo el segundo y cuarto pises más poblados de la región los cuales registran tasas bajas en acceso a internet. Mientras tanto Perú, registra un uso de internet de 70\%, resultado positivo teniendo en cuenta que es el quinto país más poblado de la Región (ASEP , 2019)

El Perú no ha sido ajeno a esta situación, pues desde el inicio de la pandemia, la economía peruana se ha visto afectada en diversos sectores económicos, según un informe del Instituto Peruano de Economía (IPE) estimó que entre el 50 y 55\% del PBI, se encontraría paralizado durante el periodo de emergencia, pues solo estaba permitida la venta de bienes esenciales, los centros comerciales han dejado de vender S/1,400 millones de soles durante los primeros 28 días del estado de emergencia. (IPE, 2020)

En cuanto al comercio online (Castro, 2017), refiere que a los consumidores les resulta más fácil y sencillo comprar a través de canal online, pues perciben un ambiente igual de atractivo y amigable al comprar en una tienda física como la virtual, por el contrario les supone mayor riesgo al efectuar pagos a través de internet, debido a frecuentes robos de información y ataques a los sistemas de seguridad, percibiendo menor riesgo al comprar en tienda física que en las online, dado por la ausencia y el contacto físico que existe en este tipo de transacciones online generando desconfianza en el consumidor.

Para (Mamani y Rosplgliosi, 2017), en un contexto nacional encontraron que los factores que determinan la intención de uso de canales online de prendas de vestir calzado y accesorios de moda, son la facilidad de uso, en lo sencillo y útil, que les resulta comprar vía online, resaltando el cumplimiento de las promesas de estas plataformas, su alto uso, le resulta beneficioso y positivo al comprar sus prendas de vestir, además recomiendan realizar capacitaciones para enseñar cómo usar las diversas herramientas de los canales de comercialización y logar que las personas que desconfían conozcan el sistema, para facilitar su uso, convirtiéndola en una herramienta útil para simplificar y ahorrar tiempo y esfuerzo conectarse con el mundo, conseguir oportunidades que les permita mayores beneficios y descuentos al comprar en línea, además de hacer notar las ventajas que ofrece la tecnología. 
Para las empresas con comercio online desarrollado o en desarrollo, los escenarios creados por el COVID-19 son importantes, indicadores que le permitirán aplicar estrategias oportunas y apropiadas en la medida que puedan superar las barreras de conectividad, disponibilidad de mercancías, plazos de entrega, crear un entorno que garantice la seguridad de las operaciones que vayan a realizar por este medio, además de lograr generar confianza en la nueva experiencia en aventurarse en el uso de canales online, se debe entender qué tipo de dispositivos utilizan, las categorías preferidas que en la mayoría de ocasiones está relacionada con los años donde no es de extrañarse que estos consumidores online sean jóvenes. (Perú Retail, 2020)

\section{Formulación de problema general y específicos}

\section{Problema general}

¿De qué manera los canales de comercialización online impactan en el incremento de las ventas en tiempos de COVID-19, Lima Metropolitana 2020?

\section{Problemas específicos}

- ¿De qué manera los canales de comercialización online impactan en la intención de compra en tiempos de COVID-19, Lima Metropolitana 2020?

- ¿De qué manera los canales de comercialización online impactan en el riesgo de compra en tiempos de COVID-19, Lima Metropolitana 2020?

- ¿De qué manera los canales de comercialización online impactan en el nivel de gasto en tiempos de COVID-19, Lima Metropolitana 2020?

- ¿De qué manera los canales de comercialización online impactan en la satisfacción de compra en tiempos deCOVID-19, Lima Metropolitana 2020?

\section{Marco teórico}

\section{Variable independiente: Los Canales de comercialización online}

Es un modelo basado en la compra/venta mediante el uso de Internet, anteriormente algunos negocios solo realizaban ventas de productos por este medio y no por tienda física, sin embargo, ahora se pueden adquirir de forma física como virtual. (Castaño y Jurado, 2016) Consiste en ofertar bienes y servicios, haciendo uso de las diferentes herramientas que ofrece el internet a través de aplicaciones creadas en un entorno digital, y el uso de éstas como estrategia de marketing en las empresas vanguardistas, para fomentar acciones de interacción comercial entre el cliente y la empresa, encaminadas al nuevo entorno digital sin necesidad de acercarse a un establecimiento. (Martínez y Rojas, 2016) en la cual el cliente puede realizar la adquisición de un producto o servicio, haciendo uso de una página web sin importar el lugar donde se encuentre además de integrar sus sistemas con otras empresas, con la finalidad de elevar su eficiencia y la disminución de sus costos, donde las transacciones personales y comerciales se trasladan a medios computarizados vía online, así como las diferentes actividades en la operación. (Somalo 
Peciña, 2017) Estas operaciones son realizadas por medios electrónicos haciendo uso de dispositivos como teléfonos móviles, tablets, computadoras, con acceso a internet.

El comercio electrónico es una herramienta que ayudaría al flujo de efectivo al realizarse ventas más efectivas, reducir costos en las transacciones, buscando aumentar la eficiencia y eficacia en los procesos y operaciones organizacionales (Alderete, Jones y Motta, 2016) ideadas como parte de las estrategias que deben implementarse para obtener la máxima ventaja que ofrece el internet.

\section{Variable dependiente: Incremento de las ventas}

Lograr ventas exitosas ha sido siempre el objetivo de toda unidad social denominada empresa, que se crea con la finalidad de obtener ganancias a través de todo bien o servicio sea tangible o intangible que puede ser vendido y percibir un ingreso económico al realizar un intercambio comercial, pues en el uso de tiendas virtuales, los clientes pueden informarse e incluso adquirir productos de manera más rápida que en una tienda física, haciendo uso de una tarjeta o de dinero electrónico, y lo más importante, que esta acción puede ser realizada las 24 horas y desde el lugar donde se encuentre el consumidor. (Carrasco Fernández, 2014) Contando con las herramientas necesarias y las debidas medidas de seguridad.

La venta tiene un doble objetivo, la de satisfacer una necesidad que requiere el cliente y la de generar ingresos por la realización de ella; así mismo, cabe resaltar que la venta es un conglomerado de acciones establecidas para la adquisición de un bien o servicio. (Carrasco Fernández, 2014) Considerando la venta como un contrato en el cual el vendedor está obligado a entregar un bien o servicio al comprador, obteniendo a cambio una compensación monetaria.

\section{Teoría de la segmentación de mercado:}

La segmentación de mercados, es una secuencia en la que se logra identificar determinados consumidores con características de compras similares, quienes componen un segmento o grupo, con la finalidad de establecer ofertas diferenciadas para ellos, que suplan sus necesidades y cumplan con sus expectativas. (Galicia Pérez y López Rodríguez, 2015) al segmentar el mercado permitirá ofrecer estrategias adecuadas, para elevar las ventas de acuerdo a las necesidades de la población a quien nos dirigiremos, al conocer las principales necesidades de este, y satisfacerlas totalmente, mediante el uso de herramientas de gestión para la delimitación de conjunto de clientes o consumidores, los cuales cuentan con necesidades comunes, el objetivo es de ofrecer información necesaria o promociones específicas para este grupo de clientes. (Martínez Valverde J. F., 2018) creando submercados de tamaños más reducidos y uniformes utilización, edad, estilo de vida como variable para realizar dicha segmentación.

\section{Teoría de la facilidad de uso:}

La facilidad de uso se define como la impresión del cliente en base a su gestión de compra mediante cualquier herramienta digital en la web, y que ésta requiera de un mínimo 
esfuerzo. (Peña-García, 2018) Determinada por la rapidez con la que un usuario puede desenvolverse en la compra de un bien o servicio en la web.

La empresas deben maximizar la facilidad de uso y la comodidad en el proceso de compra de los usuarios buscando fidelizarlo y como una variable ligada directamente a la satisfacción del consumidor (Iconaru, 2012) referida a la practicidad con la que un cliente pueda realizar sus compras mediante una página web u otra herramienta digital.

\section{Teoría de la categoría de producto:}

La categorización de un producto o servicio es un instrumento de organización, técnica de éxito adoptada por Amazon desde 1994, al incorporar categorías de productos como nueva forma de comprar con un gran catálogo de productos, diversas categorías, precios bajos y un sistemas logístico que permite disponer del producto adquirido en 24 horas (Osorio, 2016) permite clasificar los bienes o servicios de tal forma que posibilite la agrupación en un listado, y este sea más accesible para el consumidor, ordenadas y distribuidas de manera eficaz para que el cliente pueda adquirirlas de manera práctica.

\section{Teoría de la confianza del consumidor:}

La confianza es de suma importancia al momento de realizar compras online, y más cuando el producto tiene un alto riesgo de adquisición, pues por medio de las compras electrónicas no se puede establecer una relación física con el vendedor ni con el bien. (Rovira, 2017).

La confianza con la que cuente el cliente al momento de comprar por internet es vital ya que de ello dependerá que el proceso de compra llegue al final.

\section{Teoría de la intención de compra:}

Cuando un cliente ingresa al local así sea solo para mirar algún producto, demuestra su intención de compra, pero dependerá del vendedor concretar la venta. (Herrera, 2016) Pues como en las páginas web, si el cliente navega por la página es porque tiene intención de comprar, depende de que ésta sea fácil de acceder y capte la atención del consumidor.

En el contexto del marketing la intención de compra distingue dos tipos de decisión, las compras planificadas y las compras por impulso con intención de comprar algún bien o servicio (Santesmases, Merino, Sachez y Pintado, 2011).

\section{Teoría del riesgo de compra:}

Relacionado con la confianza que percibe el consumidor. Toda decisión involucra un riesgo, por lo que el cliente opta por las que cuenten menor riesgo para él o para su dinero. (Rovira, 2017) Toda decisión tiene un riesgo, y para que este disminuya debemos tener previa información de la empresa antes de realizar una transacción económica. 
El riesgo percibido por los consumidores en las transacciones en línea, son una de las barreras del comercio electrónico, donde la interceptación de datos personales captadas por criminales cibernéticos son usados en actos criminales y fraudulentos (J.P Morgan, 2013)

La participación del consumidor en las compras vía online dependerá de si el riesgo que percibe al realizar esta acción es elevado.

\section{Teoría del nivel de gasto:}

Statista proyecta que casi 2000 millones de persona adquieren bienes y/o servicios online durante el 2019, donde más de 155,5 millones provendrían de América latina, lo que significa que una de cada cuatro habitantes de la región actualmente hace uso de los medios digitales para la adquisición de algún producto o servicio. (BlackSip, 2019)

Se concluye que la mayoría de los habitantes en América Latina adquieren productos y/o servicios mediante los canales de comercialización online.

\section{Teoría de la satisfacción compra:}

La satisfacción percibida por el consumidor a la compra realizada es la apreciación final por parte de este al concluir con la adquisición de un bien o servicio. Al momento en que el consumidor analiza el producto comprado, también evalúa la experiencia concebida durante el proceso de compra. (Eslava, 2012) Es establecer una escala de satisfacción basándose en cómo el bien o servicio adquirido a través de la web, cumplen o superan sus expectativas, en ello influye en los beneficios del producto y el precio de éste.

\section{Antecedentes}

\section{Nacionales}

(Rodas Alarcón, 2017), en su estudio "El Efecto del Comercio Electrónico en el proceso de comercialización de artesanías en la empresa Industria Prada", inicia con la necesidad de implementar nuevos modelos de negocio acorde con las tendencias tecnológicas de vanguardia, lo cual sirvió para establecer el objetivo general del presente, que es determinar el efecto en el proceso de comercialización a través del uso del comercio electrónico en la empresa Industrias Prada. Con apoyo del diseño cuasi experimental del enfoque cuantitativo con pre y post prueba, espaciados en un periodo de tiempo de cinco meses. Los resultados reflejan que no hubo variación significativa en cuanto al nivel ventas en el periodo analizado, pero sí hubo una variación positiva significativa respecto a la cobertura de publicidad que se incrementó exponencialmente, teniéndose este registro en la cantidad de visitas visualizadas en la herramienta Google analytics.

(Figueroa Piscoya y Silva Rengifo, 2016), en su investigación titulada "Plataforma de comercio electrónico para optimizar el proceso de ventas de la mediana y pequeña empresa en la región Lambayeque". Con el objetivo de: Desarrollar una Plataforma de comercio electrónico para el proceso de ventas de la mediana y pequeña empresa de la región Lambayeque, en la que 
analiza, diseña y desarrolla una Plataforma de Comercio Electrónico que permitirá a las MYPES de la región Lambayeque una manera fácil, rápida y eficiente de ofrecer sus productos en línea, llegar a más clientes y mejorar el proceso de ventas, como también promover la venta física en sus locales. Concluye que la plataforma de comercio electrónico es una solución que integra los procesos de pedido y venta de productos terminados; con la interacción de las redes sociales se captarían más clientes hacia los diferentes negocios afiliados a esta plataforma de comercio electrónico.

(Morán Marchan y Cabrera Galarza, 2017), desarrollaron un estudio sobre el “Análisis de los factores que influyen en el nivel de satisfacción de los compradores de linio en América Latina" donde quieren dar a conocer el origen y las estrategias aplicadas en el comercio electrónico, así como factores que influyen de manera positiva y negativa de la satisfacción del cliente, y su nivel de compra, donde analizó a la empresa Linio y sus operaciones en México, Chile y Perú, teniendo como base el nivel de satisfacción de los compradores online. Basándose en un análisis cuantitativo y cualitativo de fuentes primarias y secundarias, con encuestas a altos ejecutivos de Linio, revisión bibliográfica e indicadores del mismo Linio para medir el nivel de satisfacción. Donde se concluyó que influyen directamente en el nivel de satisfacción de los consumidores online: la calidad de productos, el tiempo de entrega y las diferentes formas de medios de pago que ofrezcan los diferentes comercios online; y que la cadena logística también influye de manera directa en el nivel de satisfacción de los compradores online, el diseño de la página web, así mismo el tiempo de respuesta a una pregunta del consumidor influye directamente en la decisión de compra y por último se comprobó que la cantidad de medios de pago ofrecidos por Linio influye en el nivel de satisfacción del comprador online.

\section{Internacionales}

(Gómez Aceldas, 2018), realizó una tesis titulada "Plan estratégico de marketing digital 2018-2019 para la empresa control - D en la ciudad de Cúcuta. Norte de Santander”, su naturaleza de investigación es cualitativo y cuantitativo, pues realizaron un análisis de sus preferencias y cualidades de los clientes para generar las estrategias de marketing digital al crear tendencia en la web, además a través de datos estadísticos y gráficas se llegaron a conclusiones para la toma de decisiones. El objetivo de esta investigación es de diseñar un plan estratégico de marketing digital 2018 -2019 para la empresa Control-D. Por otra parte, el enfoque de la investigación es de naturaleza cualitativa y cuantitativa, para el desarrollo de la presente investigación, se consideró un diseño de campo y bibliográfico. El primero se aplicó a la empresa virtual Control-D donde a través de una entrevista se determinaron las fortalezas y debilidades que presenta la organización y el segundo se utilizó para recopilar información por fuentes documentales e información que permitieron identificar las condiciones del entorno que la afectan. Donde concluyen indicando que la empresa Control-D es una organización que cuenta con una propuesta de valor definida y un mercado objetivo al cual dirigir sus esfuerzos, pero no cuenta con una estructura de marketing digital por lo que se le dificulta llegar a sus clientes a través de las plataformas online utilizadas actualmente. El plan de marketing digital debe enfocarse en la planeación estratégica ya que de esta forma se ayudan a cumplir los objetivos que se han propuesto. 
(Sánchez Alzate y Montoya Restrepo, 2016), realizaron una investigación sobre los "Factores que afectan la confianza de los consumidores por las compras a través de medios electrónicos", su metodológica utilizada para alcanzar el objetivo propuesto se centra en la recolección, procesamiento y análisis de información secundaria relacionada con el marketing, la Internet, el comercio electrónico y la confianza; se identifican los factores que influyen en la confianza por las compras en línea. El análisis se presenta a través de una descripción conceptual, y la utilización de técnicas de representación de información como tablas y figuras, se demuestra cómo los consumidores sienten menos temor y más predisposición a la compra por medios electrónicos cuando la confianza es mayor. Gracias a la revisión sistemática de la bibliografía se pudo analizar el impacto de la confianza en las compras en línea y determinar que los factores más influyentes en ella son: la reputación del vendedor, el riesgo percibido, la privacidad de los datos, la seguridad en la transacción, la asociación, y el género del comprador. Se concluye, finalmente, que gracias a estos aspectos los consumidores se inclinan con mayor facilidad a desarrollar sus compras online.

(Castro Tuñas, 2017), realizó una investigación relacionada a la Distribución Física y Online: Análisis de la Categoría de Producto como Factor Determinante en la elección del Canal con el objetivo de analizar cómo los consumidores perciben la compra de productos a través de diferentes canales de distribución para dos categorías de producto distintas: alimentación y productos de electrónica. Con este estudio empírico se pretende realizar un análisis comparativo entre los diferentes canales de distribución para cada categoría de producto, ya que, según la literatura previa sobre el tema, parece lógico que existan diferencias en cuanto a la aceptación de un canal de distribución u otro, para estas categorías de producto. La metodología de investigación utilizada es la primera parte del estudio empírico, la cual tiene como objetivo realizar un análisis descriptivo de las percepciones de los consumidores sobre las diferentes canales de distribución para cada categoría de producto, se ha realizado una distribución de frecuencias relativas, luego analizará los valores medios y desviaciones típicas. Se realizó un análisis comparativo entre la distribución física y distribución online para las diferentes categorías de producto: alimentación y electrónica. Lo que queda claro, es que la distribución física y online no compiten, sino que son canales de distribución complementarios. Por lo tanto, cualquier empresa que quiera ser competitiva hoy en día, debería ofrecer a sus usuarios ambas posibilidades de compra.

\section{Metodología}

La presente investigación cuenta con un enfoque cuantitativo, según (Hernández Sampieri, Fernández Collado y Baptista Lucio, 2014), se caracteriza por seguir un conjunto de procesos que tiene una secuencia y además se puede probar; partiendo de ello, podemos obtener información actual para dar respuesta a nuestro problema general, siguiendo un procedimiento ordenado.

Respecto al tipo de investigación, esta es básica ya que se orienta a buscar nuevos conocimientos y campos de investigación sin un fin práctico específico e inmediato, tal cual lo indica (Rodríguez Arainga, 2011); aplicado en nuestro trabajo nos permite apoyarnos con un marco teórico para las variables de la investigación. 
Esta investigación es de nivel explicativo, basado según (Hernández Sampieri, Fernández Collado y Baptista Lucio, 2014), en describir conceptos, fenómenos o de establecer las relaciones que hay entre estos conceptos; en nuestro caso vamos a explicar la manera en que los canales de comercialización online impactan en el incremento de ventas en tiempos de COVID19, Lima Metropolitana 2020.

El diseño de investigación es de tipo no experimental, según (Hernández Sampieri, Fernández Collado y Baptista Lucio, 2014), es cuando las variables no tienen ningún tipo de manipulación intencional, se evalúan, observan y analizan tal cuál su contexto natural. Este a su vez puede dividirse en transversal donde recolecta datos en un solo momento.

En cuanto a la población, ésta es parte de un universo, la cual nos sirve para poder recopilar los datos que van a definir quiénes serán parte de la unidad de análisis, es decir, específicamente hacía quien va a ir orientada nuestra investigación, tal como lo indica (Hernández Sampieri, Fernández Collado y Baptista Lucio, 2014), para esta investigación, la población son habitantes de Lima Metropolitana, que han utilizado algún canal de comercialización online, y esta población no es conocida o finita, su magnitud es desconocida.

La muestra según (Hernández Sampieri, Fernández Collado y Baptista Lucio, 2014), es parte de la población a quién se le aplicará el siguiente proceso de investigación, como puede ser una encuesta, en base a esos resultados se contrastará la hipótesis; para ello, se escogerá a los habitantes de la ciudad de Lima Metropolitana, ambos sexos y que se encuentren en el rango de 18 a 55 años, obteniendo un total de 6672000 habitantes que cumplen con esta característica de selección. Se establece un nivel de confianza de $95 \%$ y un margen de error de $6 \%$, aplicando la fórmula de población conocida, obtuvimos como resultado la cantidad de 267 personas.

En cuanto al muestreo, éste será aleatorio simple, ya que, según (INEGI, 2011), es la modalidad del muestreo probabilístico donde cada integrante de la población tiene la misma probabilidad de ser seleccionado, basándonos en los criterios de selección adecuados para el tema planteado y así integrar la muestra.

Por otro lado, en cuanto a la técnica (Gil Pascual, 2016), hace relación a los medios técnicos utilizados para el registro de observaciones o tratamiento de la información; en la presente investigación se aplicó la técnica de encuesta, para la recolección de datos.

En cuanto al instrumento, (Fábregues, Meneses, Rodriguez y Paré, 2016), menciona que el cuestionario es el instrumento de forma estándar para la recolección de información en el trabajo de campo; por ello es que realizamos un cuestionario en escalamiento de Likert como instrumento y así poder evaluar las variables de investigación, donde el encuestado responderá una serie de preguntas basadas en los indicadores planteados.

La validez según (Sáenz López, Gorjón Gómez, Gonzalo Quiroga y Díaz Barrado, 2012), se refiere a la importancia de la relación entre los conceptos teóricos con los indicadores, para hallar la validez de nuestro proyecto, requerimos del juicio de 02 expertos los cuales analizaron cada pregunta de nuestro instrumento, y luego de ponderar el resultado obtuvimos que el $\mathrm{V}$ de Aiken es de 0,94 lo que nos indica que el presente trabajo tiene un alto índice de validez, ya que

Esta obra se comparte bajo la licencia Creative Common Atribución-No Comercial 4.0 International (CC BY-NC 4.0) 
según la teoría el valor va desde 0,00 hasta 1,00 siendo este la mayor magnitud de validez posible.

La confiabilidad se enfoca en el grado de precisión con la que un determinado instrumento facilita la información, tal cual lo menciona (Sáenz López, Gorjón Gómez, Gonzalo Quiroga, y Díaz Barrado, 2012). Para establecer una consistencia interna el Alfa de Cronbach se debe encontrar entre 0,70 y 0,90; en nuestro estudio contamos con un Alfa de Cronbach de 0,912 , es decir, que cuenta con una alta consistencia entre sus variables.

\section{Resultados y discusiones}

\section{Tabla 1}

Pruebas de normalidad

\begin{tabular}{clccccc}
\hline & \multicolumn{3}{c}{ Kolmogorov-Smirnov ${ }^{\mathrm{a}}$} & \multicolumn{3}{c}{ Shapiro-Wilk } \\
\cline { 2 - 7 } & Estadístico & $\mathrm{gl}$ & Sig. & Estadístico & $\mathrm{gl}$ & Sig. \\
\hline $\begin{array}{c}\text { Canales de } \\
\text { comercialización }\end{array}$ &, 072 & 267 &, 002 &, 986 & 267 &, 011 \\
\hline Incremento de ventas &, 090 & 267 &, 000 &, 963 & 267 &, 000 \\
\hline
\end{tabular}

a. $\quad$ Corrección de significación de Lilliefors

Fuente: Base de datos IBM SPSS

La muestra es 267, al ser mayor de 50 se analiza el resultado de Kolmogorov-Smirnova

Como la significancia < 0.05, se acepta la Ha es decir los datos no siguen distribución normal y por lo tanto el estadístico que se trabajará es la correlación no paramétrica de Chi cuadrado.

\section{Comprobación de hipótesis}

- Hipótesis general:

Ho: Los canales de comercialización online NO impactan significativamente en el incremento de las ventas en tiempos de COVID-19.

Ha: Los canales de comercialización online SI impactan significativamente en el incremento de las ventas en tiempos de COVID-19.

Criterio de aceptabilidad:

Si la sig <0.05, se acepta la Ha, es decir, que los canales de comercialización online SÍ influye significativamente en el incremento de venta. 


\section{Tabla 2}

Tabla cruzada entre Canales de comercialización e Incremento de ventas

\begin{tabular}{lllllll}
\hline & & & \multicolumn{3}{c}{ Incremento de ventas } & \multirow{2}{*}{ Total } \\
\cline { 3 - 6 } & & & Bajo & Medio & Alto & \\
\hline \multirow{3}{*}{$\begin{array}{c}\text { Canales de } \\
\text { Comercialización online }\end{array}$} & \multirow{2}{*}{ Bajo } & Recuento & 37 & 16 & 1 & 54 \\
\cline { 2 - 6 } & \multirow{2}{*}{ Medio } & \% del total & $13,9 \%$ & $6,0 \%$ & $0,4 \%$ & $20,2 \%$ \\
\cline { 2 - 6 } & & Recuento & 12 & 132 & 15 & 159 \\
\cline { 2 - 6 } & \multirow{2}{*}{ Alto } & \% del total & $4,5 \%$ & $49,4 \%$ & $5,6 \%$ & $59,6 \%$ \\
\cline { 3 - 6 } & & Recuento & 0 & 20 & 34 & 54 \\
\cline { 3 - 6 } Total & \multirow{2}{*}{ \% del total } & $0,0 \%$ & $7,5 \%$ & $12,7 \%$ & $20,2 \%$ \\
\hline & & Recuento & 49 & 168 & 50 & 267 \\
\cline { 3 - 6 } & & $\%$ del total & $18,4 \%$ & $62,9 \%$ & $18,7 \%$ & $100,0 \%$ \\
\hline
\end{tabular}

Fuente: Base de datos IBM SPSS

Tabla 3

Pruebas de chi-cuadrado

\begin{tabular}{lllc}
\hline & Valor & gl & $\begin{array}{l}\text { Significación asintótica } \\
\text { (bilateral) }\end{array}$ \\
\hline Chi-cuadrado de Pearson & $191,516^{\mathrm{a}}$ & 4 &, 000 \\
\hline Razón de verosimilitud & 161,298 & 4 &, 000 \\
\hline Asociación lineal por lineal & 121,909 & 1 &, 000 \\
\hline N de casos válidos & 267 & & \\
\hline
\end{tabular}

Nota: 0 casillas $(0.0 \%)$ han esperado un recuento menor que 5. El recuento mínimo esperado es 9.91.

Fuente: Base de datos IBM SPSS

La sig. < 0.05, se acepta la Ha, es decir los canales de comercialización online SI impactan significativamente en el incremento de las ventas en tiempos de COVID-19, Lima Metropolitana 2020.

Por lo tanto, cuando se utilicen los canales de comercialización online, habrá un incremento en las ventas en tiempos de COVID-19. Es decir, el incremento de ventas está asociado a los canales de comercialización online.

La investigación presenta una hipótesis general, donde se puede evidenciar que existe una relación positiva entre los canales de comercialización online y el incremento de las ventas en 
tiempo de COVID-19, ya que el nivel de significancia fue de $0,000<0.05$; es decir si hay un alto impacto entre los canales de comercialización online y el incremento de ventas.

Nuestros resultados guardan relación con los expresado por Rodas (2017), donde se realizó una investigación de diseño cuasi experimental del enfoque cuantitativo para determinar el efecto en el proceso de comercialización a través del comercio electrónico, donde los resultados en ambos estudios resultaron positivos, ya que concluyen en que la utilización de los canales de comercialización online para comprar nos permite tener mayor acceso a más clientes y por lo tanto mayor posibilidad de incrementar las ventas.

Se considera la teoría de Matute et al. (2012), donde expresan que el proceso del comercio electrónico permite que las empresas ofrezcan y vendan sus productos o servicios en un mercado global permitiendo llegar a una mayor cantidad de posibles clientes.

- $\quad$ Hipótesis específica $\mathrm{N}^{\circ} 01$ :

Ho: Los canales de comercialización online NO impactan significativamente en la intención de compra en tiempos de COVID-19.

Ha: Los canales de comercialización online SI impactan significativamente en la intención de compra en tiempos de COVID-19.

Criterio de aceptabilidad:

Si la sig <0.05, se acepta la Ha, es decir que los canales de comercialización online SI influye significativamente en la intención de compra.

\section{Tabla 4}

Pruebas de chi-cuadrado

\begin{tabular}{lllc}
\hline & Valor & gl. & $\begin{array}{l}\text { Significación asintótica } \\
\text { (bilateral) }\end{array}$ \\
\hline Chi-cuadrado de Pearson & $206,259^{\mathrm{a}}$ & 4 &, 000 \\
Razón de verosimilitud & 177,063 & 4 &, 000 \\
Asociación lineal por lineal & 133,187 & 1 &, 000 \\
\hline N de casos válidos & 267 & & \\
\hline
\end{tabular}

Nota: 0 casillas $(0,0 \%)$ han esperado un recuento menor que 5. El recuento mínimo esperado es 8,90. Fuente: Base de datos IBM SPSS

La sig. < 0.05, se acepta la Ha, es decir los canales de comercialización online SI impactan significativamente en la intención de compra en tiempos de COVID-19, Lima Metropolitana 2020. 
De acuerdo a la primera hipótesis, los resultados guardan relación con los obtenidos por Castro (2017), quien en su investigación en España respecto a la distribución física y online para la compra de productos de alimentación y productos de electrónica", encontró que tanto para los productos de alimentación, como para la electrónica, permiten afirmar que la intención de compra de los consumidores es mayor en la distribución física, es decir que prefieren realizar sus compras en una tienda física.

En la presente investigación se aplicó una encuesta a una muestra representativa de la población, los cuales son comparables con los obtenidos por Castro (2017), los resultados guardan relación con los obtenidos en la presente investigación, ya que ambos estudios concluyen que los encuestados manifiestan tener un bajo uso de los canales de comercio online en distintos escenarios sociales, culturales y en entornos restrictivos de libre tránsito.

En cuanto a lo mencionado por Herrera (2016), indica que en un negocio cuando un cliente ingresa al local así sea solo para mirar o preguntar el precio del algún producto en especial, demuestra su intención de compra, ya que quizás su subconsciente lo hizo ingresar, pero dependerá del vendedor concretar la venta.

- Hipótesis específica $\mathrm{N}^{\circ} 02$ :

Ho: Los canales de comercialización online NO impactan significativamente en el riesgo de compra en tiempos de COVID-19.

Ha: Los canales de comercialización online SI impactan significativamente en el riesgo de compra en tiempos de COVID-19.

Criterio de aceptabilidad:

Si la sig <0.05, se acepta la Ha, es decir que los canales de comercialización online SI influye significativamente en el riesgo de compra.

\section{Tabla 5}

Pruebas de chi-cuadrado

\begin{tabular}{llll}
\hline & Valor & gl & $\begin{array}{l}\text { Significación asintótica } \\
\text { (bilateral) }\end{array}$ \\
\hline Chi-cuadrado de Pearson & $86,452^{\mathrm{a}}$ & 4 &, 000 \\
\hline Razón de verosimilitud & 80,295 & 4 &, 000 \\
\hline Asociación lineal por lineal & 64,109 & 1 &, 000 \\
\hline N de casos válidos & 267 & & \\
\hline
\end{tabular}

Nota: 0 casillas $(0,0 \%)$ han esperado un recuento menor que 5. El recuento mínimo esperado es 9,30.

Fuente Base de datos IBM SPSS 
La sig. $<0.05$, se acepta la Ha, es decir los canales de comercialización online SI impactan significativamente en el riesgo de compra en tiempos de COVID-19, Lima Metropolitana 2020.

Respecto a la segunda hipótesis específica, se puede evidenciar que existe un impacto positivo entre los canales de comercialización online y el riesgo de compra, ya que el nivel de significancia fue de $0,000<0.05$ que lo demuestra.

Nuestros resultados guardan relación con la investigación científica de Sánchez (2015), el cual analiza los factores que afectan la confianza en el consumidor al realizar compras por internet, la metodología que utilizaron fue la de recolectar, procesar y analizar información bibliográfica; donde se muestra que los factores más influyentes en la confianza son la reputación del vendedor, el riesgo percibido, privacidad de datos y la seguridad en la transacción; en ambos estudios se comprueba que hay una relación positiva entre los canales de comercialización online y el riesgo de compras, ya que el cliente al ingresar a una plataforma online para comprar, ésta le genera confianza a través de diferentes elementos influyentes para aminorar el riesgo de compra y por ende aumentar la confianza percibida.

Rovira (2017) manifiesta que el riesgo de compra está relacionado con la confianza que percibe el consumidor, donde el cliente desarrolla ciertas tácticas para disminuirlo optando por aquellas que representen un riesgo menor; además Hair (2011) nos dice que existen diferentes tipos de riesgo, como el financiero, social y psicológico.

- Hipótesis específica $\mathrm{N}^{\circ} 03$ :

Ho: Los canales de comercialización online NO impactan significativamente en el nivel de gasto en tiempos de COVID-19.

Ha: Los canales de comercialización online SI impactan significativamente en el nivel de gasto en tiempos de COVID-19.

Criterio de aceptabilidad:

Si la sig <0.05, se acepta la Ha, es decir que los canales de comercialización online SI impactan significativamente en el nivel de gasto.

\section{Tabla 6}

Pruebas de chi-cuadrado

\begin{tabular}{lllc}
\hline & Valor & gl & $\begin{array}{l}\text { Significación asintótica } \\
\text { (bilateral) }\end{array}$ \\
\hline Chi-cuadrado de Pearson & $81,029^{\mathrm{a}}$ & 4 &, 000 \\
\hline Razón de verosimilitud & 74,853 & 4 &, 000 \\
\hline Asociación lineal por lineal & 63,119 & 1 &, 000 \\
\hline N de casos válidos & 267 & & \\
\hline
\end{tabular}

Notas: 0 casillas $(0,0)$ han esperado un recuento menor que 5 . El recuento mínimo esperado es 11,93. Fuente: Base de datos IBM SPSS 
La sig. $<0.05$, se acepta la Ha, es decir los canales de comercialización online SI impactan significativamente en el nivel de gasto en tiempos de COVID-19, Lima Metropolitana 2020.

Además, en referencia a la tercera hipótesis, nuestros resultados son comparables con los obtenidos por Castro (2017), quien afirma que los consumidores perciben en canal de venta online como un canal de distribución que ofrece precios más adecuados y que pueden pagar la mayoría de los consumidores, en comparación con las tiendas físicas, en un contexto diferentes en cuanto a aspectos culturales y restrictivos.

Por consiguiente, se considera la teoría de BlackSip (2019) el cual proyecta que casi 2000 millones de persona adquieren bienes y/o servicios online durante el 2019, donde más de 155,5 millones provendrían de América Latina, lo que significa que una de cada cuatro habitantes de la región actualmente hace uso de los medios digitales para la adquisición de algún producto o servicio.

- $\quad$ Hipótesis específica $\mathrm{N}^{\circ} 04$ :

Ho: Los canales de comercialización online NO impactan significativamente en la satisfacción de compra en tiempos de COVID-19.

Ha: Los canales de comercialización online SI impactan significativamente en la satisfacción de compra en tiempos de COVID-19.

Criterio de aceptabilidad:

Si la sig <0.05, se acepta la Ha, es decir que los canales de comercialización online SI influye significativamente en la satisfacción de compra.

\section{Tabla 7}

Pruebas de chi-cuadrado

\begin{tabular}{lllc}
\hline & Valor & gl & $\begin{array}{l}\text { Significación asintótica } \\
\text { (bilateral) }\end{array}$ \\
\hline Chi-cuadrado de Pearson & $96,905^{\text {a }}$ & 4 &, 000 \\
\hline Razón de verosimilitud & 93,933 & 4 &, 000 \\
\hline Asociación lineal por lineal & 76,442 & 1 &, 000 \\
\hline N de casos válidos & 267 & & \\
\hline
\end{tabular}

Nota: 0 casillas $(0,0 \%)$ han esperado un recuento menor que 5. El recuento mínimo esperado es 9,91. Fuente: Base de datos IBM SPSS 
Concluyendo con la sig. De $<0.05$, se acepta la Ha, es decir los canales de comercialización online SI impactan significativamente en la satisfacción de compra en tiempos de COVID-19, Lima Metropolitana 2020.

En cuanto a la cuarta hipótesis específica, se puede evidenciar la satisfacción en la compra vía Internet, por lo tanto, los canales de comercialización online si impactan significativamente en la satisfacción de compra, ya que el nivel de significancia fue de $0,000<$ 0,05 .

Nuestros resultados se comparan con los de Moran y Cabrera (2017) donde realizan una estudio a la empresa Linio Perú, Chile y México, basándose en la satisfacción del cliente, cuyo análisis es cuantitativo y cualitativo de fuentes primarias y secundarias, determinando que hay una relación directa en una compra online y la satisfacción de compra, donde se ve reflejada principalmente a través de la calidad del producto, el tiempo de entrega, y las diferentes formas de pago que pueda ofrecer el canal online; así mismo, se concluye que ambos estudios se relacionan de manera positiva, ya que se demostró que la satisfacción del cliente, no sólo está en sí en el producto, sino otros factores como la atención a la solución de un inconveniente y la rapidez en la respuesta que se le da al cliente.

De acuerdo con lo expresado, se toma en consideración la teoría de Eslava (2012), quien indica que la satisfacción percibida por el consumidor se define como la apreciación final al concluir con la adquisición de un bien o servicio, además no sólo evalúa el bien, sino la experiencia durante el proceso de compra.

\section{Conclusiones}

En cuanto a lo abordado con anterioridad, las estadísticas demuestran que los canales de comercialización online, impacta significativamente con el incremento de las ventas en tiempos de COVID-19, ya que el nivel de significancia fue de $0,00<0,05$. El coeficiente correlación no paramétrico de Chi cuadrado fue de 0,00 demostrando dicha relación. Por lo tanto, el uso de los canales de comercialización online si tiene un impacto significativo en el incremento de las ventas en tiempos de COVID-19.

Se identifica que, si existe un impacto significativo entre los canales de comercialización online y la intención de compra, debido a que el nivel de significancia fue de $0,00<0,05$. El coeficiente correlación no paramétrico de Chi cuadrado fue de 0,00 demostrando que existe una considerable relación. Es decir que el uso de los canales de comercialización online si tiene un gran impacto en la intención de compra.

De este modo, las estadísticas demuestran que los canales de comercialización online impactan positivamente con el riesgo de compra, ya que el nivel de significancia fue de $0,00<$ 0,05. El coeficiente correlación no paramétrico de Chi cuadrado fue de 0,00 lo cual indica una dependencia fuerte y alta. Es decir que el uso de los canales de comercialización online si tiene un gran impacto en el riesgo de compra. 
Se concluye finalmente que, según los datos analizados, los canales de comercialización online impactan significativamente con el nivel de gasto, ya que el nivel de significancia fue de $0,00<0,05$. El coeficiente correlación no paramétrico de Chi cuadrado fue de 0,00 lo cual demuestra la fuerte dependencia entre variable y dimensión. Es decir, que el uso de los canales de comercialización online si tiene un impacto positivo en el nivel de gasto.

Se identificó finalmente que, según los datos obtenidos, los canales de comercialización online impactan significativamente con la satisfacción de compra, ya que el nivel de significancia fue de $0,00<0,05$. El coeficiente correlación no paramétrico de Chi cuadrado fue de 0,00 demostrando que existe un impacto relevante. Esto significa que, el uso de los canales de comercialización online si tiene un gran impacto en la satisfacción de compra.

\section{Referencias bibliográficas}

Alderete, M. V., Jones, C., y Motta, J. (2016). Gestión estratégica de tecnologías de información y comunicación y adopción del comercio electrónico en Mipymes de Córdoba, Argentina. Estudios Gerenciales, 32 (138), 4 - 13. Obtenido de www.icesi.edu.co: https://www.icesi.edu.co/revistas/index.php/estudios_gerenciales/article/view/2187

Armas, M., y Colmenares, M. (2009). Educación para el desarrollo de la cultura tributaria. REDHECS, 6(4), 141-160. Obtenido de https://dialnet.unirioja.es/descarga/articulo/2937210.pdf

Arriaga, G., Reyes, M., Olives, J., y Solórzano, V. (2017). Análisis de la cultura tributaria: Impuesto a la renta par personas naturales no obligadas a llevar contabildad, Provincia de Santa Elena. Ciencias Pedagógicas e Innovación UPSE, 5(3), 118-127. doi:DOI http://dx.doi.org/10.26423/rcpi.v5i3.192

ASEP . (Agosto de 2019). Reporte de Industria El Ecommerce en Peru 2019. Obtenido de asep.pe: https://asep.pe/wp-content/uploads/2019/08/Reporte-de-industria-deleCommerce-Peru-2019-eBook.pdf

ASEP. (2019). Reporte de industria del Ecommerce Peru 2019. Digital Business Partners, 5-7. Recuperado el 2020, de https://asep.pe/wp-content/uploads/2019/08/Reporte-deindustria-del-eCommerce-Peru-2019-eBook.pdf

Barrio Carrasco, J. (2017). La influencia de los medios sociales digitales en el consumo. La función prescriptiva de los medios sociales en la decisión de compra de bebidas refrescantes en España. Universidad Complutense de Madrid, Madrid, España. Recuperado el 2020, de https://eprints.ucm.es/42339/1/T38702.pdf

Bedoya, A., y Vásconez, B. (2010). Entendiendo la moral tributaria en Ecuador. Revista Fiscalidad(5), 91-132. Obtenido de https://cef.sri.gob.ec/pluginfile.php/16836/mod_page/content/64/5_3.pdf 
Berlanga, V., Rubio, M., y Vilá, R. (2013). Cómo aplicar árboles de decisión en SPSS. Revista d'Innovació i Recerca en Educació, 6(1), 65-79. Obtenido de http://diposit.ub.edu/dspace/bitstream/2445/43762/1/618361.pdf

BID. (2020). Los Desafíos del Comercio Electrónico para las Pyme. Banco Interamericano de Desarrollo, 11-17. Recuperado el 2020, de https://publications.iadb.org/publications/spanish/document/Los-desafios-del-comercioelectronico-para-las-PyME-Principales-claves-en-el-proceso-de-digitalizacion.pdf

BlackSip. (2019). Reporte de Industria: El e-commerce en Perú. BlackSip, 4. Recuperado el 2020, de https://content.blacksip.com/ebook-reporte-de-industria-el-ecommerce-en-peru2019

Brian S. Cole, J. H. (2018). Eleven quick tips for architecting biomedical informatics workflows with cloud computing. PLOS Computational Biology Education.

Cabezas Mejía, E. D., Andrade Naranjo, D., y Torres Santamaría, J. (2018). Introducción a la metodología de la investigación científica. (1 ed.). Ecuador: Comisión Editorial de la Universidad de las Fuerzas Armadas ESPE. Recuperado el 2020, de http://repositorio.espe.edu.ec/bitstream/21000/15424/1/Introduccion\%20a\%201a\%20Met odologia\%20de\%20la\%20investigacion\%20cientifica.pdf

Carrasco Fernández, S. (2014). Técnicas de venta. España: Editorial Paraninfo. Recuperado el 2020, de https://books.google.com.pe/books?id=kscUBQAAQBAJ\&printsec=frontcover\&hl=es

Carrasco Fernández, S. (2014). Venta Online. España: Editorial Paraninfo. Recuperado el 2020, de https://books.google.com.pe/books?id=2pGLBAAAQBAJ\&printsec=frontcover\&hl=es

Carrasco, M. (2010). La ciudadania fiscal: algunas reflexiones. Revista Fiscalidad(5), 11-66. Obtenido de https://cef.sri.gob.ec/pluginfile.php/16836/mod_page/content/64/5_2.pdf

Castaño, J. J., \& Jurado, S. (2016). Comercio electrónico. Madrid, España: EDITEX. Recuperado el 2020, de https://books.google.com.pe/books?id=dJ1cDAAAQBAJ\&printsec=frontcover

Castaño, j. J., y Jurado, S. (2016). Comercio Electronico . Madrid, España: Editex.

Castro Tuñas, A. (2017). Distribución física y online: Análisis de la categoría de producto como factor determinante en la elección de canal. Universidad de Coruña, Coruña, España. Recuperado el 2020, de https://ruc.udc.es/dspace/bitstream/handle/2183/19800/CastroTu\%C3\%B1as\%20_Alba_ TFG_2017.pdf?sequence $=2$

Castro, T. A. (2017). Distribución física y online: Análisis de la categoría de producto como factor determinante en la elección de canal. Distribución física y online: Análisis de la 
categoría de producto como factor determinante en la elección de canal. Universidad Da Coruña - Facultad de Economia y Empresa, La Coruña.

CVE. (2019). Common Vulnerabilities and Exposures. Obtenido de cve.mitre.org/

David G. Rosado, C. B.-M. (s.f.). La Seguridad como una asignatura indispensable. XVI Jornadas de Enseñanza Universitaria de la Informática.

Delgado, J. V. (2015). ¡Planificando Estratégicamente! California, USA: Windmills International Editions Inc. Recuperado el 2020, de https://books.google.com.pe/books?id=Zg4sCgAAQBAJ\&printsec=frontcover

Digital Commerce 360. (30 de Julio de 2020). Los ingresos de Amazon en el segundo trimestre de América del Norte aumentan un $43 \%$ a medida que las ventas de comestibles en la web se triplican. Obtenido de Digital Commerce 360:

https://www.digitalcommerce360.com/article/amazon-sales/

Eslava, J. (2012). Pricing: nuevas estrategias de precios (3 ed.). Madrid, España: Editorial ESIC. Recuperado el 2020, de https://books.google.com.pe/books?id=R0IT5x9M0MC\&printsec=frontcover\&hl=es

Fábregues, S., Meneses, J., Rodriguez, D., \& Paré, M.-H. (2016). Técnicas de investigación social y educativa. Editorial UOC. Recuperado el 2020, de https://www.researchgate.net/profile/Julio_Meneses/publication/303346000_Tecnicas_de _investigacion_social_y_educativa/links/577b45ab08aec3b743357c8a/Tecnicas-deinvestigacion-social-y-educativa.pdf

Fernández, M., y Calero, J. (2011). Los efectos no monetarios de la educación, Análisis a partir del consumo de los hogares. Revista de Educación, 419-442. Obtenido de http://www.revistaeducacion.mec.es/re355/re355_18.pdf

Figueroa Piscoya, E. N., y Silva Rengifo, R. R. (2016). Plataforma de comercio electrónico para optimizar el proceso de ventas de la mediana y pequeña empresa en la región Lambayeque. Universidad Nacional Pedro Ruiz Gallo, Lambayeque, Perú. Recuperado el 2020, de http://repositorio.unprg.edu.pe/bitstream/handle/UNPRG/473/BC-TES4234.pdf? sequence $=1 \&$ is Allowed $=y$

Foundation, O. (2017). OWASP Top 10 - 2017 Los diez riesgos más críticos en Aplicaciones Web. Obtenido de www.owasp.org

Galicia Pérez, L. A., \& López Rodríguez, F. (2015). Entorno e información de mercados (1 ed.). España: Editorial Ideas Propias. Recuperado el 2020, de https://books.google.com.pe/books?id=URXwCAAAQBAJ\&printsec=frontcover

García-Peñalvo, F. J. (2018). Obtenido de https://repositorio.grial.eu/bitstream/grial/1228/1/07rep.pdf

Garzón, P. A. (2010). DragonJar. Obtenido de https://www.dragonjar.org/seguridadinformatica-un-reto-para-la-ingenieria-del-software-o-una-necesidad.xhtml

Esta obra se comparte bajo la licencia Creative Common Atribución-No Comercial 4.0 International (CC BY-NC 4.0) 
Gil Pascual, J. A. (2016). Técnicas e instrumentos para la recogida de información. Madrid, España: Editorial ISBN. Recuperado el 2020, de https://books.google.com.pe/books?id=ANrkDAAAQBAJ\&printsec=frontcover

Gómez Aceldas, N. (2018). Plan estratégico de marketing digital 2018-2019 para la empresa control - D en la ciudad de Cúcuta Norte de Santander. Universidad Libre de Colombia, Cúcuta, Colombia. Recuperado el 2020, de https://repository.unilibre.edu.co/bitstream/handle/10901/11482/Tesis\%20Nicolas.pdf?se quence $=1 \&$ is Allowed $=y$

Guamán, D., Guamán, F., Jaramillo, D., y Sucunuta, M. (2017). Implementation of techniques and OWASP security recommendations to avoid SQL and XSS attacks using J2EE and WS-Security. 2017 12th Iberian Conference on Information Systems and Technologies (CISTI).

Hernández Ramos, E. M., y Hernández Barrueco, L. C. (2018). Manual del comercio electrónico (1 ed.). Barcelona, España: Editorial Marge Books. Recuperado el 2020, de https://books.google.com.pe/books?id=_Dd8DwAAQBAJ\&printsec=frontcover

Hernández Sampieri, R., Fernández Collado, C., y Baptista Lucio, P. (2014). Metodología de la investigación (6 ed.). D.F., México: Editorial McGraw-Hill. Recuperado el 2020, de http://observatorio.epacartagena.gov.co/wp-content/uploads/2017/08/metodologia-de-lainvestigacion-sexta-edicion.compressed.pdf

Herrera, H. (2016). No me vedas ¡Ayúdame a comprar! (1 ed.). D.F., México: Editorial Sélector. Recuperado el 2020, de https://books.google.com.pe/books?id=ntJmCwAAQBAJ\&printsec=frontcover\&hl=es\&s ource $=$ gbs_ge_summary_r\&cad $=0$

Iconaru, C. (2012). A Decomposed Model of Consumers' Intention to Continue Buying Online. Economic Insights - Trends and Challenges, 64(4), 58-69.

INEGI. (2011). Diseño de la muestra en proyectos de encuesta. D.F., México: Editorial INEGI. Recuperado el 2020, de https://books.google.com.pe/books?id=h77ODwAAQBAJ\&printsec=frontcover

Iniestra Frias, L. A. (2015). Maestría en ciencias en estudios interdisciplinarios para pequeñas y medianas empresas. Implementación de mercadotecnia digital a una empresa de chocolate gourmet. Instituto Politécnico Nacional, D.F., México. Recuperado el 2020, de https://www.repositorionacionalcti.mx/autor/Luis+Angel+Iniestra+Frias

IPE. (20 de Abril de 2020). INFORME IPE IV: IMPACTO DEL COVID-19 EN LA ECONOMÍA PERUANA. Obtenido de ipe.org.pe: https://www.ipe.org.pe/portal/informe-ipe-ivimpacto-del-covid-19-en-la-economia-peruana/

J.P Morgan. (2013). Cybercrime: This Is War. TREASURY SERVICES, 1-9.

Kendall, K., y Kendall, J. (2011). Análisis y diseño de sistemas. México: Pearson Educación.

Esta obra se comparte bajo la licencia Creative Common Atribución-No Comercial 4.0 International (CC BY-NC 4.0) 
Llinás Solano, H., y Rojas Álvarez, C. (2017). Estadística descriptiva y distribuciones de probabilidad (21 ed.). Barranquilla, Colombia: Editorial Universidad del Norte. Recuperado el 2020, de https://books.google.com.pe/books?id=43haDwAAQBAJ\&printsec=frontcover

Lopez, C. (2012). Efecto de la educación sobre el delito: evidencia para Argentina. (Tesis de maestría, Universidad Nacional de La Plata). Obtenido de http://sedici.unlp.edu.ar/bitstream/handle/10915/24608/Documento_completo__.pdf?sequ ence $=1$

López, F. (2012). Incidencia de los conocimientos sobre tributación y el cumplimiento de las obligaciones tributarias entre el pequeño comerciante del Barrio el Beaterio en el Sector de Guajaló. (Trabajo de grado). Universal Central del Ecuador, Quito, Ecuador.

Mamani, S. Y., y Rosplgliosi, G. P. (2017). Estudio de los Factorres que determian la intención de uso del canal onlne de las prendas de vestir calzado y accesorios de moda en la ciudad de Tacna 2017. Estudio de los Factorres que determian la intención de uso del canal onlne de las prendas de vestir calzado y accesorios de moda en la ciudad de Tacna 2017. Neumann Business School, Tacna.

Martin, P. (2018). Teletrabajo y comercio electrónico. España: Editorial Aula Mentor. Recuperado el 2020, de https://books.google.com.pe/books?id=tvVKDwAAQBAJ\&printsec=frontcover

Martínez Valverde, J. F. (2018). Sistema de información de mercados (1 ed.). España: Editorial Paraninfo. Recuperado el 2020, de https://books.google.com.pe/books?id=Xf1NDwAAQBAJ\&printsec=frontcover

Martínez Valverde, J. F., y Rojas Ruiz , F. (2016). Comercio electrónico (1 ed.). Madrid, España: Paraninfo. Recuperado el 2020, de https://books.google.com.pe/books?id=nCylDAAAQBAJ\&printsec=frontcover\&hl=es\&s ource $=$ gbs_ge_summary_r\&cad $=0$

Mathkour H., A. G. (2008). A Risk Management Tool for Extreme Programming. IJCSNS International Journal of Computer Science and Network Security, 8(8), 326-333.

Mendoza, F., Palomino, R., Robles, J., y Ramírez, S. (2016). Correlación entre cultrua tributaria y educación tributaria universitaria: Caso Unversidad Estatal de Sonora. Revista Global de Negocios, 4(1), 61-76. Obtenido de ftp://ftp.repec.org/opt/ReDIF/RePEc/ibf/rgnego/rgn-v4n1-2016/RGN-V4N1-2016-5.pdf

Ministerio de Economía y Finanzas. (2017). Cifras del Presupuesto General del Estado 2018. Obtenido de https://www.finanzas.gob.ec/wpcontent/uploads/downloads/2017/11/Proforma_2018_para_Asamblea.pdf

Morales, N., Méndez, M., y Aguilera, O. (2005). Cultura tributaria y conribuyentes: datos y aspectos metodológicos. Fermentum. Revista Venezolana de Sociología y Antropología, 15(44), 332-352. Obtenido de http://www.redalyc.org/articulo.oa?id=70504404 
Morán Marchan, E., y Cabrera Galarza, Z. Y. (2017). Análisis de los factores que influyen en el nivel de satisfacción de los compradores de Linio en América Latina. Análisis de los factores que influyen en el nivel de satisfacción de los compradores de Linio en América Latina. Universidad Peruana de Ciencias Aplicadas, Lima, Perú. Recuperado el 2020, de https://repositorioacademico.upc.edu.pe/bitstream/handle/10757/621954/Cabrera_GZ.pdf ?sequence $=5 \&$ is Allowed $=\mathrm{y}$

Osorio, V. (29 de Abriñ de 2016). Expansion Economia Digital. Obtenido de expansion.com: https://www.expansion.com/economiadigital/companias/2016/07/08/577ea714e5fdea4c028b4627.html

Peña-García, N. (2018). La adopción de las tiendas electrónicas en una economía emergente. Bogotá, Colombia: CESA. Recuperado el 2020, de https://books.google.com.pe/books?id=5PmFDwAAQBAJ\&printsec=frontcover \&hl=es\& source $=\mathrm{gbs} \_g e \_s u m m a r y \_r \& c a d=0 \# \mathrm{v}=$ onepage $\& \mathrm{q} \& \mathrm{f}=$ false

Pérez, P., \& Souza, A. (2013). Como quantificar os aspectos qualitativos nas escalas de classificação de resultados NOC para etiquetas psicossocioculturais. Revista da Escola de Enfermagem da USP, 47(3), 728-735. doi:DOI: 10.1590/S0080-623420130000300029

Perú Retail. (30 de Abril de 2020). Los retos del comercio electrónico para hacer frente al coronavirus. Obtenido de peru-retail.com: https://www.peru-retail.com/retos-delcomercio-electronico-para-hacer-frente-al-coronavirus/

Quintanilla, J. (2012). La universidad en la cultura tributaria. RETOS. Revista de Ciencias de la Administración y Economía, 2(3), 105-114. Obtenido de http://www.redalyc.org/pdf/5045/504550953007.pdf

Rodas Alarcón, F. (2017). Efecto del Comercio Electrónico en el Proceso de Comercialización de Artesanías en la Empresa Industrias Prada. Efecto del Comercio Electrónico en el Proceso de Comercialización de Artesanías en la Empresa Industrias Prada. Universidad Nacional José María Arguedas, Andahuaylas, Apurímac, Perú. Recuperado el 2020, de http://repositorio.unajma.edu.pe/bitstream/handle/123456789/265/Fredy_Rodas_Tesis_Ti tulo_2017.pdf?sequence $=1 \&$ isAllowed $=\mathrm{y}$

Rodríguez Arainga, W. (2011). Guía de investigación científica. Lima, Perú: UCH. Recuperado el 2020, de http://repositorio.uch.edu.pe/bitstream/handle/uch/23/rodriguez_arainaga_walabonso_gui a\%20_investigacion_cientifica.pdf?sequence $=1 \&$ isAllowed=y

Rovira, J. (2017). Persona, no consumidor: Antropología, neurociencia y hormonas para el marketing. Madrid, España: ESIC. Recuperado el 2020, de https://books.google.com.pe/books?id=6ptMDwAAQBAJ\&printsec=frontcover\&hl=es\& source $=$ gbs_ge_summary_r\&cad $=0$ 
Ruiz, G. (9 de 10 de 2018). Los 10 Principales Riesgos de Seguridad según OWASP - Parte I. Obtenido de https://blog.sucuri.net/espanol/2018/10/los-10-principales-riesgos-deseguridad-segun-owasp-parte-i.html

Sáenz López, K. A., Gorjón Gómez, F. J., Gonzalo Quiroga, M., y Díaz Barrado, C. M. (2012). Metodología para investigaciones de alto impacto en las ciencias sociales. Madrid, España: DYKINSON, S.L. Recuperado el 2020, de https://books.google.com.pe/books?id=Cg3dBAAAQBAJ\&printsec=frontcover

Sánchez Alzate, J. A., y Montoya Restrepo, L. A. (2016). Actores que afectan la confianza de los consumidores por las compras a través de medios electrónicos. Actores que afectan la confianza de los consumidores por las compras a través de medios electrónicos.

Universidad Nacional de Colombia - Sede Medellín, Medellín, Colombia. Recuperado el 2020, de http://rcientificas.uninorte.edu.co/index.php/pensamiento/article/viewFile/8809/8594

Santesmases, M., Merino, M. .., Sachez, J., y Pintado, T. (2011). Fundamenos del Marketing. Pirmide.

Sarmiento, B. (2014). La cultura tributaria en el Ecuador. Observatorio de la economía Latinoamericana(201). Obtenido de http://www.eumed.net/cursecon/ecolat/ec/2014/cultura-tributaria.html

Schnoeller G., M. L. (2016). A strategy based on knowledge acquisition for management of requirements risks on distributed XP development. Revista lbérica de Sistemas y Tecnologías de Información(20), 18-33. doi:10.17013/risti.20.18-33

SEI. (2010). CMMI para Desarrollo, Versión 1.3. Mejora de los procesos para el desarrollo de mejores productos y servicios. EE.UU.: Technical Report, Software Engineering Institute.

SGI. (2014). The CHAOS Manifesto. Obtenido de The Standish Group International: https://www.projectsmart.co.uk/white-papers/chaos-report.pdf

SGI. (2015). Standish Group 2015 Chaos Report. Obtenido de https://www.infoq.com/articles/standish-chaos-2015

Somalo Peciña, I. (2017). El comercio electrónico: Una guía completa para gestionar la venta online (1 ed.). Madrid, España: ESIC. Recuperado el 2020, de https://books.google.com.pe/books?id=T1dqDwAAQBAJ\&printsec=frontcover

Sommerville, I. (2005). Ingeniería del Software. Pearson Educación.

Soto, M. (2016). La cultura tributaria: una contribución para la construcción colectiva del desarrollo sustentable. Novum Scientiarum, 2(1), 46-56. Obtenido de http://www.ecoambienteydesarrollo.org/revista/ojs/index.php/novum/article/view/62/cult uratributaria 
SRI. (2018). Estadísitcas generales de recaudación. Obtenido de http://www.sri.gob.ec/web/guest/estadisticas-generales-derecaudacion?p_auth=KeAzH2ym\&p_p_id=busquedaEstadisticas_WAR_BibliotecaPortle t_INSTANCE_EVo6\&p_p_lifecycle=1\&p_p_state=normal\&p_p_mode=view\&p_p_col_ id=column-1\&p_p_col_count=2\&_busquedaEstadisticas_

UCACUE. (2016). Acuerdo de cooperación interinstitucional entre la UCACUE y el SRI. [Acta convenio]. Archivo UACUE.

Vondran, A. (2015). Metodologías ágiles de gestión de proyectos. Obtenido de https://www.linkedin.com/pulse/metodolog\%C3\%ADas-\%C3\%A1giles-degesti\%C3\%B3n-proyectos-andre-vondran/

Voutssas, J. (2010). Preservación documental digital y seguridad informática. Investigación bibliotecológica. 